\title{
Strangulation als szenische Handlung. Ein Fallbericht über einen Pseudosuizidversuch im Verlauf einer NREM- Parasomnie
}

\author{
Omid Amouzadeh-Ghadikolai (D) · Laura Pascale-Scharmüller · Andreas Baranyi · Michael Lehofer · Michael Saletu
}

Eingegangen: 19. März 2018 / Angenommen: 18. Juni 2018 / Online publiziert: 10. Juli 2018

(c) Der/die Autor(en) 2018

\begin{abstract}
Zusammenfassung Es ist allgemein bekannt, dass schlafwandelnde Personen komplexe, szenische Verhaltensmuster zeigen, welche mit nicht-intendierten Konsequenzen einhergehen. Nicht selten kann es dabei zu Verletzungen des Betroffenen kommen. Sporadisch wurde aber auch ein bizarres selbst- oder fremdgefährdendes Verhalten besonderen Ausmaßes beschrieben, welches von der Tötung des Lebensgefährten bis hin zum akzidentellen (Pseudo-)Suizid reicht.

Wir berichten in diesem Artikel von einem 28-jährigen Patienten, der eines Nachts unvermittelt den Versuch unternahm, sich mit einer Peitsche zu erhängen, und diskutieren die Gründe, die dafür sprechen, dieses bizarre Verhalten als szenische Handlung im Verlauf einer NREM-Parasomnie zu interpretieren, sowie die Schwierigkeiten, die sich in der Beweisführung ergeben.
\end{abstract}

O. Amouzadeh-Ghadikolai $(\bowtie) \cdot$ M. Lehofer

Abteilung für Psychiatrie und Psychotherapie III, Standort Süd, Landeskrankenhaus Graz Süd/West, Wagner Jauregg Platz 1, 8053 Graz, Österreich omid.amouzadeh-ghadikolai@kages.at

O. Amouzadeh-Ghadikolai · L. Pascale-Scharmüller · M. Saletu

Bereich für Schlafmedizin, Standort Süd, Landeskrankenhaus Graz Süd/West, Wagner Jauregg Platz 1, 8053 Graz, Österreich

O. Amouzadeh-Ghadikolai

Universitätsklinik für Medizinische Psychologie und Psychotherapie, Medizinische Universität Graz, Auenbruggerplatz 3, 8036 Graz, Österreich

\footnotetext{
A. Baranyi

Universitätsklinik für Psychiatrie und Psychotherapeutische Medizin, Medizinische Universität Graz,

Auenbruggerplatz 3, 8036 Graz, Österreich
}

Schlüsselwörter Pseudosuizid · Schlafwandel · Parasomnie · Polysomnographie

\section{Strangulation as scenic behavior. A case report of a pseudosuicide in the course of a NREM- parasomnia}

Summary It is generally accepted, that sleepwalkers show complex behaviors leading to non-intended consequences. It is not unusual that these persons are thereby injured. However, sporadically a bizarre endangering of themselves or others is reported also, which ranges from homicide of the life partner to accidental (pseudo-)suicide.

In this article, we report on a 28year-old man who unexpectedly attempted to hang himself by a whip at night. We discuss the reasons, why this bizarre act should actually be taken as a scenic behavior in the course of a NREM-parasomnia, and the difficulty to proof this claim.

Keywords Pseudosuicide - Somnambulism · Parasomnia $\cdot$ Polysomnography

\section{Einleitung}

Der Somnambulismus zählt zur Gruppe der NREMParasomnien (Arousalstörungen), die komplexe motorische Störungen umfasst, die während des Schlafs oder während des Übergangs vom Wachen zum Schlafen auftreten können. Er kommt durch eine Dissoziation von Schlafen und Wachen zustande. Die partiell wachen Patienten sind dabei „wach genug“, um komplexe motorische Bewegungen auszuführen, aber „noch nicht ausreichend wach“, um adäquat antworten oder reagieren zu können. Für das partielle Erwachen liegt in der Regel eine Amnesie vor. 
Gewöhnlich treten Parasomnien in der Kindheit auf und sind hier Ausdruck des reifenden Gehirns. Sie können im Erwachsenenalter fortbestehen oder aber auch neu auftreten. Die Gesamtprävalenz der Parasomnie wird mit $4 \%$ in der Normalbevölkerung angegeben. Im Kindes- und Jugendalter beträgt die Prävalenz über $10 \%$.

Die Parasomnien werden gemäß der Empfehlung der American Academy of Sleep Medicine (AASM), die als Manual in Form der International Classification of Sleep Disorders (ICSD-3) [1] vorliegt und eine größere Differenzierung der schlafbezogenen Störungen als die ICD-10 und das DSM-5 Manual aufweist, diagnostisch in Untergruppen unterteilt (Tab. 1).

Wie aus Tab. 1 ersichtlich, werden Parasomnien je nach dem Zeitpunkt ihres Auftretens grundsätzlich nach REM- und N(on)REM-Schlaf eingeteilt. Der REM (Rapid-Eye-Movement)-Schlaf ist durch rasche Augenbewegungen und einen relaxierten Muskeltonus gekennzeichnet. Er tritt in etwa 3- bis 4-mal in der Nacht in einem Abstand von ca. 60-90 min auf. Die übrigen Schlafstadien werden als NREMSchlaf zusammengefasst. Die Einteilung parasomnischer Phänomene nach der Art der Schlafstadien beruht auf der Erkenntnis, dass das Aufwachen aus diesen mit unterschiedlichen Bewusstseinszuständen verbunden ist [2].

Arousalstörungen aus dem NREM-Schlaf scheinen häufig mit einer Enthemmung „grundlegender Triebe" (wie Nahrungsaufnahme, Sexualtrieb und Aggression) einherzugehen. Man nimmt an, dass zentrale Mustergeneratoren Instinktbewegungen erzeugen, die im Wachzustand durch den präfrontalen Kortex inhibiert worden wären. Das Verhalten während dieser Episoden ist nicht der äußeren Situation angemessen, weshalb sie oftmals als „psychogen“ bedingt oder als „halluzinatorische Phänomene“ verkannt werden [3].

Aus Tab. 1 wird auch deutlich, dass sich die Schlafmedizin mit zahlreichen Phänomenen beschäftigt, welchen bei psychopathologischen Erwägungen eine hohe differenzialdiagnostische Relevanz zukommen

Tab. 1 Einteilung der Parasomnien nach ICSD-3

\begin{tabular}{|c|c|}
\hline \multicolumn{2}{|c|}{ Einteilung der Parasomnien } \\
\hline \multirow{4}{*}{$\begin{array}{l}\text { Arousal-Störungen } \\
\text { aus dem NREM-Schlaf } \\
\text { heraus: }\end{array}$} & Schlaftrunkenheit \\
\hline & Schlafwandeln (Somnambulismus) \\
\hline & Pavor nocturnus \\
\hline & Schlafbezogene Essstörung \\
\hline \multirow{3}{*}{$\begin{array}{l}\text { Mit dem REM-Schlaf } \\
\text { assoziierte } \\
\text { Parasomnien: }\end{array}$} & REM-Schlaf-Verhaltensstörung \\
\hline & Rezidivierte isolierte Schlaflähmung \\
\hline & Albträume \\
\hline \multirow[t]{5}{*}{ Andere Parasomnien: } & Exploding-head-Syndrom \\
\hline & Schlafbezogene Halluzination \\
\hline & Enuresis nocturna \\
\hline & Parasomnien durch körperliche Erkrankung \\
\hline & $\begin{array}{l}\text { Parasomnien durch Medikamente, Drogen oder } \\
\text { andere Substanzen }\end{array}$ \\
\hline
\end{tabular}

kann. Dies trifft im Besonderen auch auf die Beurteilung der Zurechnungsfähigkeit von Handlungen $\mathrm{zu}$, bei welchen etwas oder jemand zu Schaden kam. Besonderes mediales Aufsehen erlangt dabei immer wieder die Diskussion von (Pseudo-?)Suiziden von Somnambulikern $[4,5]$, zuletzt etwa der unerwartete Suizid des aufstrebenden Designers Tobias Wong im Jahr 2010 [6].

Der folgende Fallbericht handelt von einem ganz ähnlich gelagerten Fall, nämlich einem 28-jährigen Somnambuliker, der eines Nachts unvermittelt den Versuch unternahm, sich mit einer Peitsche zu erhängen. Wir diskutieren die Gründe, die dafür sprechen, diese bizarre Handlung als szenische Handlung einer NREM-Parasomnie zu interpretieren, sowie die Schwierigkeiten, die sich in der Beweisführung ergeben.

\section{Kasuistik}

Hr. X, ein 28-jähriger Patient, wurde gemäß $\S 8$ des Unterbringungsgesetzes von der Intensivstation an die psychiatrische Abteilung überstellt. Der Patient wurde aufgrund einer Amnesie bezüglich der vergangenen Ereignisse und einer mangelnden Krankheitseinsicht vorerst im geschützten Bereich untergebracht. Allerdings wurde bereits in der Dokumentation des Erstgesprächs festgehalten, dass keinerlei depressive oder psychotische Symptomatik fassbar war. Da sich auch in der bald darauf erfolgten Fremdanamnese mit den Angehörigen kein Hinweis auf eine psychiatrische Erkrankung oder Anzeichen für eine Selbstgefährdung fand, erfolgte die Überstellung des Patienten in den offenen Bereich.

Hr. X und die Angehörigen zeigten sich ausgesprochen erschüttert und auch verwirrt bezüglich der für sie unverständlichen Ereignisse, die sich in der Nacht vor der Einweisung zugetragen haben: Er habe an diesem Abend einen Maskenball besucht und dort in rascher Folge eine beträchtliche Menge Alkohol zu sich genommen. Darum beschloss er, die Veranstaltung noch vor der mitternächtlichen Demaskierung zu verlassen. Die Trinkmenge ließ sich eigen- und fremdanamnestisch recht genau rekonstruieren: In etwa $5 \mathrm{~h}$ habe er folgende Getränke konsumiert: Zwei Gläser Sekt, sieben große Bier, einen Schnaps und ein Wodka-Mischgetränk.

Aufgrund der hohen Trinkmenge übergab er seinen Autoschlüssel der Lebensgefährtin, die noch bei einigen Arbeiten vor Ort half, und begab sich zu Fuß nach Hause. Etwas nach Mitternacht kam er zuhause an, putzte sich die Zähne, zog sich aus und ging ins Bett. Die Lebensgefährtin kam kurze Zeit später nach Hause und sprach ihn an, als er sich im Halbschlaf befand. Sie gingen hinunter in die Küche, und es folgte eine Diskussion über das Ausmaß seines Rauschzustandes. Die Diskussion war kurz und keineswegs hitzig. Daraufhin ging er wieder nach oben. Ab dem Moment, als 
er sich wieder ins Bett legte, fehlte ihm aber jedwede Erinnerung.

Die Lebensgefährtin aber wurde bald darauf auf ein lautes Bellen des Hundes aufmerksam. Als sie nachsehen ging, erstarrte sie plötzlich vor Schreck, als Hr. X mit einer Peitsche um den Hals die Stiege runtergekullert kam. Er war nicht ansprechbar. Der hinzugerufene Notarzt intubierte Hrn. X bei GSC 6 noch vor Ort und transferierte ihn ins Landesklinikum. Der toxikologische Befund um 01:55 Uhr wies 0,91 Promille Äthanol im Blut aus.

Die Erinnerung des Hrn. X setzte erst mit etwa 7:00 Uhr morgens wieder ein, als er auf der Intensivstation mit blauen Strangulationsstriemen am Hals erwachte. Die von Seiten der Polizei veranlasste Spurensicherung führte zu einem rekonstruierten Tathergang, wonach Hr. X sich mit einer Brauchtumspeitsche an einem Haken im (ans Schlafzimmer angrenzende) Arbeitszimmer, an welchem früher ein Boxsack hing, stranguliert habe. Dabei sei aber das Leder gerissen. Er habe sich daraufhin über die Stiege geschleppt und sei dort zu Sturz gekommen.

\section{Psychiatrische Diagnostik und Abklärung}

Weder in der Eigen-, noch in der Fremdanamnese fand sich aus psychopathologischer Sicht ein Hinweis für eine Suizidalität oder eine psychiatrische Grunderkrankung. Von den 10 Risikofaktoren für Suizidalität der SAD PERSONS Scale [7] wies Hr. X lediglich den alkoholisierten Zustand auf. Er zeigte sich durchwegs in einer euthymen, ruhigen, geordneten, im Rahmen der Abklärung vielleicht leicht besorgten, psychischen Verfassung. Die beruflichen und familiären Verhältnisse waren stabil. Hinsichtlich psychiatrischer Behandlungen und Suizidversuchen zeigte sich die Anamnese bland.

Eine MRT des Schädels, ein EEG und zahlreiche psychometrische Tests führten ebenfalls zu keinem richtungsweisenden Befund.

\section{Schlafbezogene Exploration}

Die schlafbezogene Exploration förderte jedoch Schlafwandeln als Phänomen zu Tage, welches bereits in der Kindheit auftrat und immer noch auftrete. Mittlerweile falle er jedoch nur mehr etwa alle zwei Monate durch merkwürdige nächtliche Handlungen auf. Offenbar versuche er meist die Toilette zu finden, was ihm selten gelinge. Zuletzt urinierte er in den Kleiderschrank. Unlängst redete die Freundin ihn in einem solchen Zustand an, woraufhin er angespannt, gereizt und aggressiv reagierte. Als etwa 10-jähriges Kind ging er im Ferienlager in ein anderes Zimmer und wurde im oberen Bett eines Stockbettes wieder munter. In Rauschzuständen, wie sie eingangs geschildert wurden, seien solcherart Ereignisse häufiger als sonst üblich aufgetreten.
Nach kurzem stationärem Aufenthalt wurde Hr. X mit der Verdachtsdiagnose einer NREM-Parasomnie und der Empfehlung entlassen, im Schlaflabor vorstellig zu werden, einer Empfehlung, der er kurz darauf selbständig nachkam.

\section{Schlafmedizinische Diagnostik}

Im Schlaflabor kam mit der Polysomnografie (PSG) das zurzeit umfassendste Verfahren zur Messung der physiologischen Funktionen im Schlaf zur Anwendung. Hier zeigte sich eine normale Schlafeffizienz bei einer unauffälligen Schlafarchitektur, v. a. ein normaler Tiefschlafanteil mit $15 \%$ und einen REM-Schlaf mit regulärer Atonie. In der Videoaufzeichnung konnte kein eindeutiges Parasomnieereignis festgehalten werden. Die Aufwachrate war im Normbereich. Es fand sich kein Hinweis für eine schlafbezogene Atemstörung oder periodische Beinbewegungen (etwa als möglicher Trigger für Parasomnieereignisse).

Das begleitende EEG zeigte keine Zeichen einer erhöhten Erregungsbereitschaft oder epilepsietypische Potenziale. Doch konnte besonders in der ersten Nachthälfte ein cyclic alternating pattern (CAP) beobachtet werden (siehe Abb. 1). Dabei handelt es sich um Sequenzen transienter elektrokortikaler Ereignisse in regelmäßigen Intervallen, die sich von der Hintergrundaktivität abheben [8, 9]. Die genaue Quantifizierung ergab eine mit $43 \%$ deutlich erhöhte CAP Rate (Normbereich bei jungen Erwachsenen: 25-30\%).

\section{Diskussion}

Es liegt in der Natur der Sache, dass sich die diagnostische Zuordnung des in Frage stehenden nächtlichen Ereignisses sehr schwierig gestaltet und nicht mit letzter Sicherheit erfolgen kann.

Die Eigen- und Fremdanamnesen sowie der psychopathologische Befund scheinen aber gegen das Vorliegen einer psychiatrischen Grunderkrankung zu sprechen. Zwar ist es denkbar, dass im intoxikierten Zustand tatsächlich im Affekt bewusst ein Suizidversuch verübt wurde, der nun aber im Schulterschluss der gesamten Familie unter den Tisch gekehrt wurde, um z.B. die Stigmatisierung durch eine psychiatrische Diagnose zu verhindern. Doch schien uns die Betroffenheit der Beteiligten dafür zu sehr einfühlbar und nicht simuliert.

Für einen pathologischen Rauschzustand war die Alkoholmenge zu hoch und es fehlte die Tendenz zur Fremdaggression. Eine schlafbezogene hypermotorische Epilepsie (sleep-related hypermotor epilepsy) ist aufgrund der unauffälligen Befunde des Routine-EEG und der Schlaf-EEG-Ableitung im Rahmen der PSG allein durchaus nicht ausgeschlossen, doch zeigen sich bei einer solchen meist nur Sekunden dauernde, motorische Stereotypien ohne szenischen Verlauf, 


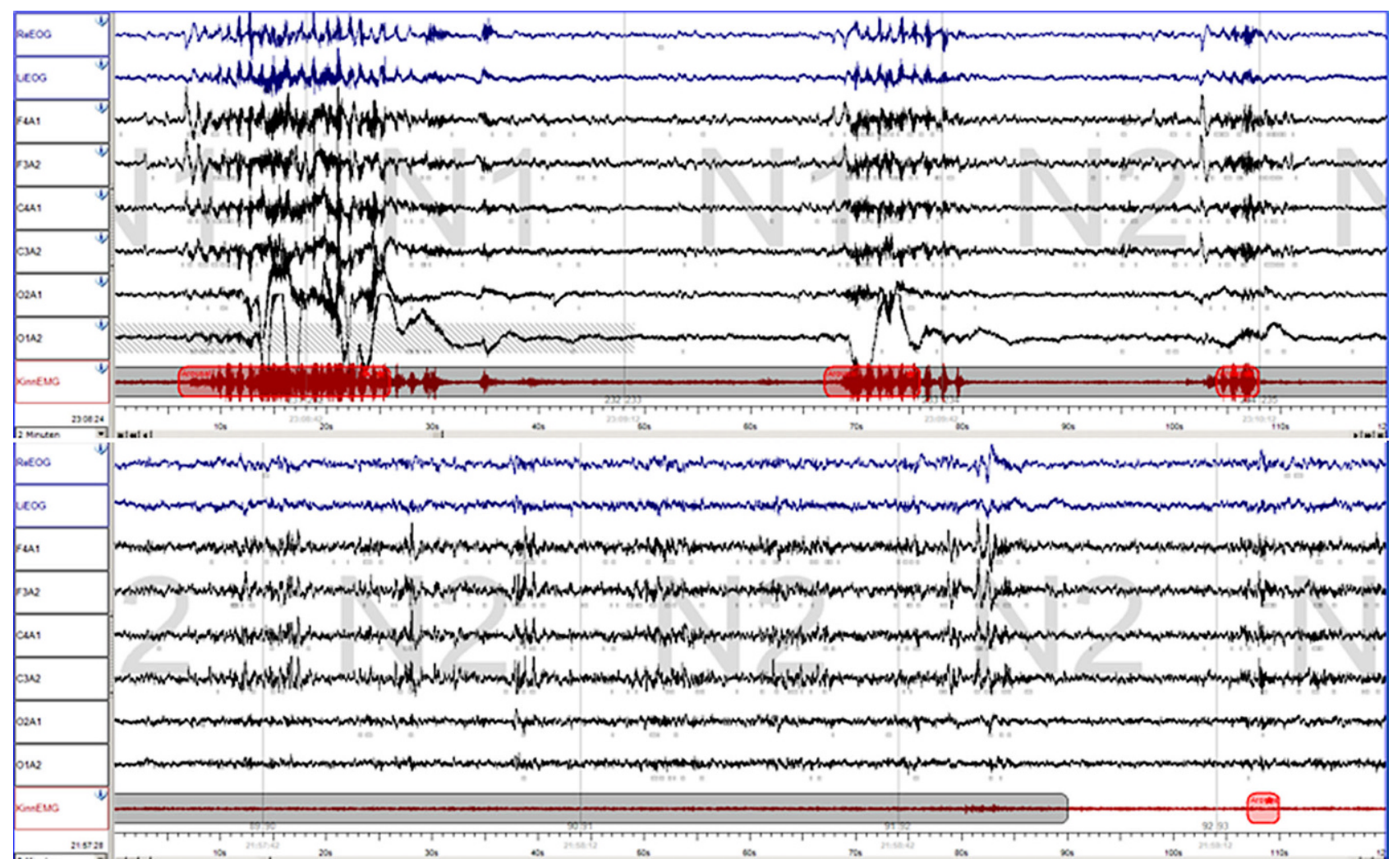

Abb. 1 Cyclic alternating pattern (CAP) in der Polysomnographie des Patienten

die zudem mit einer sehr hohen Frequenz (oft jede Nacht) auftreten [10].

Die Bizarrheit, Komplexität und Unvermitteltheit der selbstgefährdenden Handlung, welche in der ersten Nachthälfte und offenbar mit geöffneten Augen vollzogen wurde, sprechen gemeinsam mit der einschlägigen Anamnese und der retrograden Amnesie sehr stark für eine szenische Handlung im Rahmen eines Somnambulismus, der bekanntlich durch Alkoholeinfluss und Schlafentzug provoziert werden kann [2, 3]. Leider wird in der PSG nur äußerst selten ein solches Parasomnieereignis direkt aufgezeichnet. Indirekt richtungsweisend sind aber eine hypersynchrone Deltaaktivität sowie eine erhöhte cyclic alternating pattern (CAP) Rate, welche letztere auch wir in der PSG vorfanden. Sie dienen als Marker einer NREMInstabilität, die als pathophysiologisches Korrelat des Somnambulismus angesehen wird [9, 11]. Dieser Befund stützte somit die positive Anamnese hinsichtlich des Somnambulismus, wodurch jener in unseren $\mathrm{Au}$ gen zur wahrscheinlichsten Ursache der Strangulation avancierte.

In der Therapie des Schlafwandels können versuchsweise niedrigdosiert Benzodiazepine mit längerer Halbwertszeit (z. B. Clonazepam) oder verschiedene ,anekdotisch als wirksam berichtete“ [2] Antikonvulsiva eingesetzt werden [12, 13]. Als Wirkmechanismus wird eine Tiefschlafunterdrückung angenommen.
Die verhaltenstherapeutisch angewandte Vorsatzbildung sowie die Hypnose können mögliche therapeutische Interventionen darstellen [12, 14]. Es liegen aber ebenso wie für die pharmakologischen Interventionen keine Daten zum Effekt über einen längeren Zeitraum vor, weshalb keine der genannten Maßnahmen als sichere Therapieempfehlung angesehen wird $[2,3,12]$.

Aufklärung hinsichtlich provozierender Faktoren (z.B. Alkoholabusus oder Schlafentzug), Absicherung der Bettumgebung sowie die Instruktion des Bettpartners (beruhigendes „Talking Down“) haben sich bislang am effektivsten zur Verhütung einer Selbstoder Fremdverletzung erwiesen [2]. Auch im vorliegenden Fall schienen uns diese Maßnahmen am ehesten zielführend.

Zusammenfassend lässt sich festhalten, dass bei der differentialdiagnostischen Abklärung nächtlicher Störungen und Ereignisse der verschiedensten Art auch Parasomnien in Betracht gezogen werden sollten. Besonders in unklaren oder bizarren Fällen sollte neben einer gründlichen EEG-Abklärung auch eine Vorstellung in einem Schlafmedizinischen Zentrum mit neuropsychiatrischem Schwerpunkt erwogen werden. Im Falle einer entsprechenden Diagnosestellung sind Maßnahmen zur Verhütung einer Selbstoder Fremdgefährdung verhältnismäßig einfach umzusetzen. 
Danksagung Wir bedanken uns bei unserem Patienten für die freundliche Zustimmung zur Publikation dieses Fallberichts.

Funding Open access funding provided by Medical University of Graz.

Interessenkonflikt O. Amouzadeh-Ghadikolai, L. PascaleScharmüller, A. Baranyi, M. Lehofer und M. Saletu geben an, dass kein Interessenkonflikt besteht.

Open Access Dieser Artikel wird unter der Creative Commons Namensnennung 4.0 International Lizenz (http:// creativecommons.org/licenses/by/4.0/deed.de) veröffentlicht, welche die Nutzung, Vervielfältigung, Bearbeitung, Verbreitung und Wiedergabe in jeglichem Medium und Format erlaubt, sofern Sie den/die ursprünglichen Autor(en) und die Quelle ordnungsgemäß nennen, einen Link zur Creative Commons Lizenz beifügen und angeben, ob Änderungen vorgenommen wurden.

\section{Literatur}

1. American Academy of Sleep Medicine. International classification of sleep disorders. 3. Aufl. Darien: American Academy of Sleep Medicine; 2014.

2. Young P, Möddel G. Schlafwandeln und andere Non-REMParasomnien. Akt Neurol.2014;41:225-36.

3. Saletu M, Saletu-Zyhlarz GM. Schlafwandeln, Schlaftrunkenheit und Nachtschreck: die klassischen NREM-Parasomnien und ihre Differenzialdiagnoseim Erwachsenenalter. Somnologie. 2015;19:226-32.
4. Lauerma H. Fear of suicide during sleepwalking. Psychiatry. 1996;59(2):206-11.

5. Mahowald MW, Schenck CH, Goldner M, Bachelder V, Cramer-Bornemann M.Parasomniapseudo-suicide.JForensic Sci. 2003;48(5):1158-62.

6 . The Mysteries of Tobias Wong. The New York Times, 27.Juni 2010.

7. Patterson WM, Dohn HH, Bird J, Patterson GA. Evaluation of suicidal patients: the SAD PERSONS scale. Psychosomatics. 1983;24(4):343-5, 348-349.

8. Terzano MG, Parrino L, Sherieri A, Chervin R, Chokrorverty $\mathrm{S}$, et al. Atlas, rules, and recording techniques for the scoring of cyclic alternating pattern (CAP) in human sleep. Sleep Med.2001;2:537-53.

9. MoserD. Cyclicalternating pattern, Klassifikationskriterien und Bedeutung für die klinische Anwendung. Somnologie. 2015;19:46-50.

10. Tinuper P, Provini F, Bisulli F, Vignatelli L, Plazzi G, et al. Movement disorders in sleep: guidelines for differentiating epileptic from non-epileptic motor phenomena arising from sleep. Sleep Med Rev. 2007;11:255-67.

11. Guilleminault C, Kirisoglu C, da Rosa AC, Lopes C, Chan A. Sleepwalking, a disorder of NREM sleep instability. Sleep Med.2006;7:163-70.

12. Reid WH, Ahmed I, Levie CA. Treatment of sleepwalking: a controlled study. AmJ Psychother. 1981;35:27-37.

13. Reid WH, Haffke EA, Chu CC. Diazepam in intractable sleepwalking: a pilot study. Hillside J Clin Psychiatry. 1984;6:49-55.

14. Oudiette D, Constantinescu I, Leclair-Visonneau L, Vidailhet M, Schwartz S, et al. Evidence for the re-enactement of a recently learned behavior during sleepwalking. PLoS ONE. 2011;6:e18056. 